论坛.

\title{
生物多样性价值的经济学处理： 一些理论障碍及其克服
}

\author{
徐嵩龄 \\ ( 中国社会科学院环境与发展研究中心, 北京 100732)
}

摘要 : 本文首先对国际生态经济学界围绕 世界生态系统功能价值计量” 的方法学论争进行简短的评论, 接着阐述 了生物多样性价值的新的概念框架, 并据此就生物多样性价值计量中尚未得到关注的问题 (生物多样性价值的可 计算性, 价值计量方法的恰当性, 价值分量的可加性和可解析性, 价值误差测算) 提出解决思路。本文最后讨论了 两个保护经济学问题的方法学构架, 其一是生物多样性破坏的经济损失计算, 其二是生物多样性保护工程的成本 - 效益分析与成本 - 效果分析。

关键词：生物多样性，价值计量方法，可计算性，可加性，可解析性，误差测算，损失计算，成本 - 效益分析，成本 - 效 果分析

中图分类号 :Q16 文献标识码 : B 文章编号 : 1005-0094(2001)03-0310-09

\section{Economic approaches to valuing biodiversity : some theoretical barriers and relevant counter-measures}

XU Song-Ling

Center for Environment and Development Research , Chinese Academy of Social Sciences , 100732

\begin{abstract}
This paper provides a short commentary on the methodological controversy surrounding valuation of world ecosystem services. A new conceptual framework is then proposed for understanding the value of biodiversity, and, based on this, a number of issues are discussed, involving measuring the value of biodiversity, which have not yet been resolved. These are the computability of value, the validity of valuation methods, the additivity and analyticity of value sectors, and error measurement of the value. Finally the paper presents methodological frameworks for two important issues of conservation economics , one of which is estimation of the cost of biodiversity disturbance, and the other is cost-benefit analysis ( CBA ) and cost-effectiveness analysis (CEA) for conservation engineering.
\end{abstract}

Key words : biodiversity, valuation methods , computability , additivity , analyticity , error measurement , costing bio-disturbance, Cost-Benefit Analysis, Cost-effectiveness Analysis

\section{1 引言 : 炎 世界生态系统功能价值”论争谈起}

生物多样性价值的经济学处理, 是保护经济学 ( conservation economics)的基础与核心。然而，它所依托或建立的 概念与方法至今尚不具备充分的可操作性。

生物界的价值是需要通过人类的感知来表达和体现的。 因此, 利用人类社会的经济学概念和方法来处理生物界价值 问题，在一定程度上是可行的。

对于生物多样性, 人们多是从其生态功能的角度来认识 和处理它的经济价值的。从理论上讲, 生物多样性价值的经
济学计量与生态系统功能的经济学计量, 无论在概念上还是 方法上应当是一致的和相通的, 然而两者并不完全等同。这 是因为生态系统功能是一个系统的表征, 而生物多样性只涉 及生态系统的一个组成部分 (即生物部分)。因此, 如果以 生态系统功能价值来代替生物多样性价值, 那么在逻辑上是 矛盾的、不合理的，在实践上将是有害的、失败的。更何况， 关于生态系统功能价值的经济学计量至今也并非完善。这 里回顾和评论 1997 年及其后围绕世界生态系统功能价 值” 的学术论争, 或许对于认识与讨论生物多样性价值的经 济学计量会有启发性意义。 
$1997 \sim 1998$ 年的那场论争，一方是 世界生态系统功能 和自然资本的价值”一文 (Costanza et al. , 1997) 的作者 R. Costanza 为首,他们基本上是国际刊物 Ecological Economics (生态经济学) 的同仁与同道, 因而可称 生态经济学派”; 另 一方是对此文的批评者 D. Pearce, V. Smith 等人, 他们有着 充分的环境经济学研究背景, 因而可称 环境经济学派”。 两派在学术背景上的差异在于:前者的生态学背景较强而经 济学背景较弱; 而后者则相反, 经济学背景较强而生态学背 景较弱。围绕 Costanza 等人的论文，双方争论性文字多达数 篇 (Pimm , 1997 ; Sagoff , 1997 ; Pearce , 1998 ; Costanza et al. , 1998)。这里根据 Pearce 与 Costanza 等发表在 Environment 杂志上的诘难 (Pearce , 1998) 与答辩 (Costanza，1998)， 将双方的论点列于表 1 。

与任何有价值的学术论争一样, 这是没有完全的赢家与 输家的。公正地说,Pearce 等人对 Costanza 等人工作的经济 学挑剔是深刻的、有力的。只要 生态系统功能价值” 的计 量没有真正与经济学接轨, 它就不可能为经济学所接受并对 经济实践产生影响。另外, Costanza 等人的反驳在某些点上 亦具有创新性的突破(如提出世界 GNP 应将世界生态系统 功能价值包括在内, 以及坚持世界生态系统功能价值的总体 可计算性)。值得注意的是，他们的论争文字是以下述话语
宣告结束的。Pearce 说“: Costanza 等作者的工作在追求宣传 效果的同时, 是以破坏他们赖以利用的这一学科 (即经济学 一本文作者注) 的完整性为代价的”;而 Costanza 说 “"与 Pearce 教授不同, 我们不认为现已有一条给生态系统功能定 价的途径, 但是如果什么也不做, 那肯定是一个错误”。如果 Pearce 的话是对有关方法学错误的警告的话,那么 Costanza 的表白则透露出继续前进的勇气。的确, 为了成功地解决这 一问题, 除了前进, 还有什么呢? 而为了前进, 除了接受上述 警告并规避它，还有什么呢?

上述论争在研究方法学上的启示性意义在于 :在生态系 统功能价值 (包括生物多样性价值) 的经济学计量问题上, 或者广而言之, 在生态学与经济学融合的问题上, 人们往往 热衷于从正面提出各种解决问题的概念和方法, 这固然是必 要的; 但同样必要而且重要的是, 人们也需要从反面去思考 依然存在的各种障碍以及现有概念和方法中的不恰当性等 等。能否进行这种反向思考, 在新概念与方法已有相当积累 的情况下, 尤其可能会成为影响前进的主要因素。然而, 这 种反向思考往往在研究中受到忽视。

本文将对 生物多样性价值的经济学处理” 问题进行反 向思考, 将着重讨论这种价值计量的可操作性障碍。本文将 依次讨论生物多样性的各价值分量的涵义及可计算性; 各价

表 1 “世界生态系统功能价值”论争中正反双方的主要论点

Table 1 Main arguments of the two sides on the value of the World's Ecosystem Services

\begin{tabular}{|c|c|c|}
\hline 论题 & $\begin{array}{l}\text { 正方“: 生态经济学派” } \\
\text { ( Costanza 等人) }\end{array}$ & $\begin{array}{c}\text { 反方“: 环境经济学派” } \\
\text { ( Pearce 等人) }\end{array}$ \\
\hline $\begin{array}{l}1 \text { 世界生态系统功能价值的可 } \\
\text { 计算性 }\end{array}$ & 可以计算 总”价值 & $\begin{array}{l}\text { 难以计算 总”价值 或者其计算结果没有实 } \\
\text { 际意义。 }\end{array}$ \\
\hline 2 生态功能价值计量方法 & $\begin{array}{l}\text { 市场价格 } \\
\text { 准 (Pseudo) 市场价格 } \\
\text { 替代成本 }\end{array}$ & $\begin{array}{l}\text { “货币化”二原则 } \\
\text { (1) 以支付意愿 ( WTP) 表达的 消费者偏 } \\
\text { 好”。 } \\
\text { (2) 边际分析。 }\end{array}$ \\
\hline \multicolumn{3}{|l|}{3 对对方的批评 } \\
\hline \multirow[b]{2}{*}{ 1) 关于 支付意愿" } & (1) 支付意愿” 并不一定是对的, 它有很大的局限性。 & $\begin{array}{l}\text { (1)所谓的 文化价值”未能反映人们的 支 } \\
\text { 付意愿”。 }\end{array}$ \\
\hline & $\begin{array}{l}\text { (2) 世界生态系统功能价值” 可以超过现时世界的 } \\
\text { GNP, 因为后者没有将世界生态系统功能价值包括在 } \\
\text { 内。然而,一个真正有效的 GNP 是应当包括生态系统 } \\
\text { 功能价值的。 }\end{array}$ & $\begin{array}{l}\text { (2)根据 支付意愿” “, 世界生态系统功能价 } \\
\text { 值”不可能超过世界 GNP 之和。 }\end{array}$ \\
\hline \multirow[b]{2}{*}{2 ) 关于 边际分析" } & $\begin{array}{l}\text { (1)世界生态系统功能价值是一个宏观经济学问题, 而不 } \\
\text { 是微观经济学问题, 因此, 不必建立在边际分析之上。 }\end{array}$ & $\begin{array}{l}\text { (1)价格必须建立在边际分析的基础上, 它应 } \\
\text { 与物品总量的变化相联系,而不是与总量相 } \\
\text { 联系, 因此, 利用这一价格计算物品总量价 } \\
\text { 值是不对的, 或是无意义的。 }\end{array}$ \\
\hline & $\begin{array}{l}\text { (2)世界生态系统功能价值作为一个宏观量, 它与 GNP } \\
\text { 一样, 均是可以计算的。 }\end{array}$ & $\begin{array}{l}\text { (2)GNP 可以计算不等于 世界生态系统功能 } \\
\text { 价值” 可以计算。这是因为 GNP 和人造资 } \\
\text { 本的计算利用了可以观察到的市场价格, 即 } \\
\text { 反映现时供求状况的边际价格。如果这一 } \\
\text { 状况改变, GNP 与人造资本价格也随之改 } \\
\text { 变。在计算世界生态系统功能价值”时, 未 } \\
\text { 能循此路径。 }\end{array}$ \\
\hline
\end{tabular}


值计量方法的恰当性; 价值分量的可加性，可分解性; 价值计 算的误差估测。本文最后将为两类与生物多样性价值有关 的保护经济学问题提出相应的经济学处理的概念与方法构 架。这两个问题, 其一是生物多样性破坏的经济损失计算, 其二是生物多样性保护工程的投入与产出分析。

2 生物多样性价值的概念框架: 价值来源、价值空 间与表达

关于环境与自然资产的价值，人们大多或是从价值的施 予方角度来界定，如 de Groot (1992) 将 自然的价值”认定为 4 类共 37 种功能。也有从价值的使用方角度加以定义, 如 Pearce 将 环境的价值”确定为 4 类, 即直接使用价值、间接 使用价值、选择价值、存在价值 (Pearce \& Turner ,1990)。这 里试图为对生物多样性的价值认识同时也是为对生态、环 境、自然的价值认识建立一种新的概念构架，从而能将生物 多样性价值的经济学处理置于更为理性的水平上。这一节 将依次讨论生物多样性的价值来源、构成与表达。

生物多样性的价值是由三方面因素决定的。它们是:生 物多样性的功能、人类对生物多样性功能的感知、生物多样 性的存在状况。

生物多样性的价值” (value) 是与它所能提供的 功能” ( services) ${ }^{\mathbb{1}}$ 密切相关的。生物多样性的功能可以从 4 个层 次来说明。它们是:种质层次、种群层次、群落层次、生态系 统层次。其中, 种质层次的功能产生自生物个体及至基因 (DNA) 的品质和特征，种群层次的功能产生自生物群体的品 质与特征, 群落层次的功能产生自不同物种间的相互作用， 生态系统层次的功能产生自生物群落与周遭环境的相互作 用。由此可以看出, 种质层次和种群层次的功能是单一物种 型的, 而群落层次的功能是多物种复合型的, 生态系统层次 的功能则是生物群与环境因素复合型的。它们之间的这些 差异, 在对具体物种的价值进行计量时, 是必须考虑的。

生物多样性的价值” 同时也与它们对人类可感知的功 利性领域的作用有关。可以将人类可感知的功利性领域概 括为三类 经济领域 (或经济相关领域)、非经济领域 (或称 与经济无关领域)、代际转移领域。生物多样性在经济领域 的价值可以直接地或间接地与市场价格挂钩; 生物多样性在 非经济领域内的价值 (如文化、美学、精神等) 虽基本上与市 场无关，但仍通过当代人的支付意愿来表达; 生物多样性在 代际转移中的价值, 则因进一步丧失客观性 (因为不存在 未 来人”这一受体) 而不得不取决于当代人的代际伦理观。上 述感知领域的差异, 是完善生物多样性价值概念与选择计量 方法时必须考虑的。

生物多样性的价值还与生物的生存状况有关。传统资 源经济学在描述资源存在特征时,大体分为两类, 即可耗竭 型 (exhaustible) 与可再生型 (reproducible)。这里的 耗竭”, 并非严格的物质意义上的 耗竭”, 而是经济学意义上 (即
“失去经济使用价值”) 的耗竭”。上述两个概念均不适用 于生物多样性经济学。第一, 这里涉及的不是 经济使用价 值”意义上的 耗竭” 问题, 而是物种的灭绝” 问题; 第二, 在 传统的资源经济学中, 被耗竭资源将被新的资源所替代, 但 在这里, 生物物种的灭绝将既是不可逆的, 也是不可替代的。 生物物种的这种灭绝”风险及其不可逆性与不可替代性，均 是在构造生物多样性价值概念与价值计量方法时必须考虑 的。

上述影响生物多样性价值的三大要素, 实际上构成了描 述生物多样性价值的三个维度 (dimension) , 即“: 功能”维 , “感知领域”维, 和 存在状态”维。这三种 维”构成了生物 多样性的价值空间。这三种维的不同的性态组合,构成了生 物多样性的形形色色的价值分量, 也构成了生物多样性价值 的总体域谱。

在上述价值空间中, 每一种生物都可找寻到自己的价值 位置。de Groot (1992) 的价值表达方式, 只不过相应于这一 价值空间中的 功能”维, 而 Pearce (1993) 的价值表达方式 只不过相应于这一价值空间中的 感知领域” 维。前面提到 的 Costanza et al. (1998) 的世界生态系统功能及自然资本 的价值”的工作, 其价值计量范畴涉及着 功能”维中的生 态系统” “, 感知领域” 维中的 经济领域”与非经济领域”, 并且在 存在状况” 维中与 灭绝”无关。下面将看到利用这 种三维表达方式, 将会更利于对有关的生物多样性价值问题 (如本文下面要讨论的生物多样性价值的可计算性问题, 价 值计量方法的恰当性问题, 价值分值的可加性问题, 可解析 性问题, 误差测算问题等等) 进行更为理性的经济学分析。

\section{3 生物多样性价值的可计算性}

生物多样性价值的可计算性, 取决于它的各个价值分量 的可计算性。这种可计算性直接依赖于人类的认知能力。 因此, 以感知领域” 维来评价可计算性, 应是最为便捷的途 径。

在 感知领域”维的三个领域中，一般来说，属于“经济” 领域或 经济相关”领域的价值分量, 大体是可以计算的。它 们或是直接利用市场价格, 或是间接地利用市场价格( 如影 子价格、替代成本等)。属于 非经济”即 与经济无关”领域 的价值分量，因其与市场价格无关而可计算性较弱。它们主 要通过 WTP(或WTA) 等 拟市场” ( pseudo-market) 途径来计 量。尽管这些WTP 量值的可信度远不如市场价格, 但由于 它们出于价值享用者自己, 所以仍具有相当的客观性。属于 “代际转移”领域的价值分量, 是难以计算的。如果一定要从 代际伦理 (即代际公平) 角度给予量化, 那么它也仅具有象征

(1) 英语的生态学文献中的 ecological function" 与生态经济学文献中 的 ecological service”均可译为 生态功能”。两者的辞意区别在于: “ ecological function” 是与 behaviour” (行为) 相联系的, 是 行为”之 果, 是无功利倾向的; 而 ecological service” 是与 utility” (效用) 相联 系的, 是 效用”之因, 是有功利倾向的。 
性或符号性意义，这是因为它的量值不是由未来人而是由当 代人代为作出的，故而没有客观性。这也意味着对于涉及代 际事务的实际决策, 不应也不能基于“价值量”进行判断，而 应基于 伦理观”进行判断。

这里还应讨论涉及物种生存状况的价值分量的可计算 性。一个濒于灭绝的物种, 其价值应是无穷大的。这既是理 论分析的结果，又符合保护实践的需求。之所以说是理论 分析的结果”, 是因为当物种种群趋于灭绝 (即分母趋于 0) 时, 将使价格向无穷大发散。之所以说符合保护实践的需 求”, 是因为如非 无穷大”, 则意味着 生物灭绝” 在价值上 是 可替代的”，从而会大大削弱乃至取消生物保护的意义。

由上述讨论可知:并非每种价值分量都具有可计算性; 而且, 对每种价值分量给以经济学定量, 既无必要, 也未必恰 当。这样,在 可计算性”这一前提下, 本文将讨论 生物多 样性价值的经济学处理” 的有关论题时，将既不涉及 生物灭 绝”问题,也不涉及生物多样性的代际转移问题, 它只涉及生 物多样性的 4 个功能层次“经济”领域与 非经济”领域、以 及在非灭绝威胁状况下的各价值分量。

\section{4 生物多样性价值计量方法的恰当性}

生物多样性价值计量方法的选择包括两个方面:一是价 格参数的选择;二是计算途径的选择。就价格参数而言, 包 括两种: 价格 I 是以市场为基础的价格参数, 它们基本上是 市场价格或由市场价格衍生的价格; 价格 II 是以 WTP 调查 为基础的意愿型价格。就计算途径而言，包括三种: 途径 I 是正面地计算各种价值分量，途径 II 是通过生物多样性破坏 引起的价值损失，从反面进行价值计算; 途径 III 是通过对被 破坏生物多样性的恢复成本计算，来替代其价值，即替代成 本法 (Replacement Cost Approach)。对生物多样性价值计量 方法的恰当性分析, 主要是针对它们各自的价格参数选择与 计算途径选择进行的。

所谓 生物多样性价值” 应是生物多样性在其享用者心 目中的价值。以此为据，即可评价在生物多样性价值计量方 法中价格参数选择与计算途径选择的恰当性, 即其产生的计 算结果是否最接近享用者心目中的价值。

就价格参数的恰当性而言, 支付意愿型价格 (即WTP) 所反映的就是享用者心目中的价值，因而是最恰当的;而市 场价格 $(P)$ 所反映的是市场交易价格。在正常情况下，它与 支付意愿相差一个“消费者剩余” ( $C S$, 即 consumer surplus)， 即 $P=W T P-C S$ 。因此, 它所反映的价值, 低于享用者心目 中的价值。这样, 就价格参数选择对价值计量结果的影响而 言, 基于 WTP 的价值计量将大于或相等基于市场价格的价 值计量。

就计算途径的恰当性而言, 途径 I 无疑是最恰当的; 途 径 II 在大多数情况下是与途径 I 等价, 有时或超过途径 I, 因 而也是恰当的, 途径 III由于是以 恢复成本” 来测度 恢复效
益” (即价值)，而这两者实际上并不相等，因而将有损其恰 当性。一般来说, 恢复成本总是小于恢复效益的 (否则为什 么要恢复呢?)。这就是成本与效益 (即价值) 之间的不对称 性。两者差距越大, 则途径 III 的恰当性愈弱。这样, 就计算 途径选择对价值计量结果的影响而言, 基于 破坏损失” 的计 量将大于或等于 真实价值”, 大于或等于 替代成本”。

从理论上讲, 由意愿型价格参数与途径 I 相组合的价值 计量方法是最恰当的, 而以替代成本为特征的计量方法是恰 当性较差的。其他组合的恰当性则位居两者之间。然而这 种理论上的恰当性并不等于实践中的可实现性。就价格参 数选择而言, 如果所有价值都以意愿型价格计算, 将是非常 不费用有效的。而最费用有效的处理是，一方面利用已有的 市场价格, 另一方面在不具备相关的市场价格的情况下, 通 过 WTP 调查, 产生意愿型价格。这样一来, 由市场价格 + 意 愿型价格的组合是实际上较为恰当的。另外, 就计算途径选 择而言, 途径 II (即从破坏损失来计算价值) 比途径 I ( 即正 面计算价值) 更具可行性。这是因为生物多样性是先天存在 的, 在正常情况下, 它的价值往往被熟视无睹, 往往需要通过 破坏引起的扰动才能全面而清晰地显示出来。这样, 应当说 (市场价格 + 意愿型价格) 与途径 II 的组合, 是实际上最恰当 的。

现代的环境经济学和自然资源经济学已发展出一系列 价值计量方法 ( Pearce，1993), 它们都可以在不同程度上应 用于生物多样性价值分量的计算。这些方法及其价格参数 与计算途径特点,可以概括于表 2 。

根据表 2 所列各方法的价格参数和计算途径的特征, 可 以判定方法 $12,4,5,7$ 的恰当性是相似的, 只有方法 3 的 恰当性较差。这样, 在对生物多样性价值分量进行实际计算 时, 应优先从 $12,4,5,6,7$ 诸法上找寻最简易可行且费用有 效的方法，只有在不得已的情况下再选用成本替代法。

\section{5 生物多样性价值的可加性}

对于生物多样性, 人们往往不仅需要知道它的价值分 量, 同时还需要知道它的价值总量。从理论上讲, 生物多样 性价值应等于其各个价值分量之和。实际上, 事情并非如此 简单。这是涉及生物多样性价值的可加性。其中包括 4 个 问题 :I. 具有可加性的生物性多样性价值的构成; II. 如何处 理综合型价值分量, 使之具有可加性; 吕. 如果协调市场价格 与意愿型价格, 使有关的价值分量具有可加性; IV. 如何调整 替代成本之值, 使有关的价值分量具有可加性。这里将逐一 加以讨论。

\section{1 具有可加性的生物多样性价值构成}

对于生物多样性来说, 并非其所有分量都是可加的, 而 应是只有具备可计算性的价值分量才是可加的。根据第 2 节的讨论, 有两类价值分量不具有可计算性。它们是涉及 “代际转移” 的价值分量与涉及 物种灭绝” 的价值分量。前 
表 2 生物多样性价值计量方法及其价格参数和计算途径特征

Table 2 Methodological characteristics of valuing biodiversity

\begin{tabular}{|c|c|c|c|}
\hline \multirow{2}{*}{ 计量方法名称 } & \multirow{2}{*}{ 内 容 概 述 } & \multicolumn{2}{|c|}{ 方法学特征 } \\
\hline & & 价格参数 & 计算途径 \\
\hline $\begin{array}{l}\text { 市场价格法 } \\
\text { Conventional } \\
\text { Market Approach }\end{array}$ & $\begin{array}{l}\text { 直接利用市场价格, 对生物多样性的一些功能进行价值 } \\
\text { 计量 }\end{array}$ & 市场价格 & 途径 I \\
\hline $\begin{array}{l}2 \text { 剂量一响应法 } \\
\text { Dose-Response } \\
\text { Approach }\end{array}$ & $\begin{array}{l}\text { 利用市场价格或影子价格, 对生物多样性扰动所产生的 } \\
\text { 影响进行价值计量 }\end{array}$ & 市场价格 & $\begin{array}{l}\text { 途径 II 为主， } \\
\text { 途径 I 为次 }\end{array}$ \\
\hline $\begin{array}{l}3 \text { 替代成本法 } \\
\text { Replacement } \\
\text { Cost Approach }\end{array}$ & 对被破坏生物多样性的恢复成本, 进行价值计量 & 市场价格 & 途径 III \\
\hline $\begin{array}{l}4 \text { 抵御性成本法 } \\
\text { Defensive } \\
\text { Expenditure } \\
\text { Approach }\end{array}$ & $\begin{array}{l}\text { 为弥补因生物多样性破坏而丧失的功能, 所需支付的费 } \\
\text { 用 }\end{array}$ & 市场价格 & 途径 II ，III \\
\hline $\begin{array}{l}5 \text { 旅游成本法 } \\
\text { Travel Cost } \\
\text { Approach }\end{array}$ & $\begin{array}{l}\text { 为弥补因生物多样性破坏而丧失的景观和环境功能,所 } \\
\text { 需支付的旅游费用的变化 }\end{array}$ & 市场价格 & 途径 II ，III \\
\hline $\begin{array}{l}6 \text { 享用型价格法 } \\
\text { Hedonic Price } \\
\text { Method }\end{array}$ & $\begin{array}{l}\text { 通过生物多样性破坏对当地地价、工资等生活要素的影 } \\
\text { 响,进行价值计量 }\end{array}$ & 市场价格 & 途径 II ，III \\
\hline $\begin{array}{l}\text { 实际市场法 } \\
\text { Experimental } \\
\text { Market or } \\
\text { Pseudo-Market } \\
\text { Approach }\end{array}$ & $\begin{array}{l}\text { 在不具备市场价格的前提下, 通过支付意愿 (WTP) 调 } \\
\text { 查,而确定生物多样性的一些价值分值, 它包括意愿型 } \\
\text { 价格法 }(\mathrm{CVM}) \text { 和意愿型排序法 (CRM) }\end{array}$ & 意愿型价格 & 途径 I, 途径 II \\
\hline
\end{tabular}

者无法计算, 后者是无穷大。因此, 这两类价值分量不具有 可加性。Pearce 有一个关于自然资本的总经济价值 (TEV) 的 计算公式 (Pearce \& Turner)，它表示为：

$$
T E V=D U V+I U V+O V+E V
$$

式中 , $D U V$ 为直接使用价值, $I U V$ 为间接使用价值, $O V$ 为选 择价值, $E V$ 为存在价值。上式的价值分量中, 严格地说, 只 有 $D U V$ 和 $I U V$ 是可计算的; $O V$ 在涉及代内转移的选择价值 时是可计算的，但这可并入 $I U V$ 即非直接使用价值之中，而 在涉及代际转移时, 是不可计算的; $E V$, 根据其定义，反映着 人们生物伦理学、环境伦理学和代际伦理学动机, 并往往涉 及灭绝问题，因而不具有可计算性。可见，Pearce 的总经济 价值”概念只具有认识上的意义, 并没有实践的可操作性。 真正能作为总经济价值计算的是 $D U V$ 与 $I U V$ 。这样 ,Pearce 的表述才能与本文的可加性讨论一致起来。

\section{2 如何处理复合型价值分量, 使之具有可加性}

在生物多样性诸价值分量中, 有一些复合型价值分量 (详见第 2 节) ,如反映群落层次功能与反映生态系统层次功 能的价值分量。这些价值分量在用于表述群落价值与生态 系统价值时是可以直接进入计算的。另外，这些价值在反映 一个关键性物种的破坏所造成的生态损失时,也是可以直接 进入计算的, 这是因为当一个物种处于生态链网结构的关键 位置时,它的破坏会导致整个链网的倾覆。然而,在计算具 体物种的价值时, 这些复合型价值分量不能直接进入计算, 因为这些价值分量不仅包容这一物种的价值, 还包括与之发
生联系和作用的其他物种和其他系统组分的价值。这样, 为 使复合型价值分量在具体物种的价值计量中具有可加性, 必 须将这一物种在群落层次或生态功能层次的作用从相应的 复合型价值分量中分离出来。只有这种分离出的价值分量, 才对具体物种的价值具有可加性。相关的分离方法, 将在下 一节生物多样性价值的可解析性” 中加以讨论。

5.3 如何协调市场价格与意愿型价格, 使有关的价值分量 具有可加性

在经济学上, 市场价格与支付意愿是既相互联系又互不 相同的两个概念。市场价格是从卖方角度而言的, 而支付意 愿是从买方角度而言的。只有当支付意愿大于或等于市场 价格, 买卖交易才会成功。其中支付意愿与市场价格之差, 就是买方 (即消费者) 所获得的消费者剩余。这样, 当讨论各 价值分值的可加性时,基于市场价格的价格分量与基于支付 意愿的价值分量, 从概念上讲, 是不能直接相加的, 而应当对 它们作一点预处理, 使它们在价值表达方式上一致起来。或 是从支付意愿中剔除消费者剩余的影响，统一地以市场价格 处理; 或是对市场价格补入消费者剩余的影响, 统一地以支 付意愿处理。

\section{4 如何调整替代成本之值, 使有关价值分量具有可加性}

在经济学上, 成本与价值并非同一范畴的概念。价值基 本上与效益更为接近。而成本与效益则是一项经济活动的 两个侧面。对于一项成功的经济活动来说, 应当效益大于成 本, 而且越大越好。如果预期的效益小于成本, 那么这一经 
济活动根本不会发生。这样，在讨论各价值分量的可加性 时,基于成本的价值分量与基于效益的价值分量，从概念上 讲，是不能直接相加的。正确的做法只能是将基于 成本”的 价值分量，通过恰当的方法(如对成本 - 效益比的某种估 计）转化为基于 效益”的价值分量。

由上述讨论可知, 要使具有可计算性的诸价值分量都具 有可加性，必须对不同范畴的价值分量进行预处理，或对复 合型价值分量进行分解，或对市场价格与支付意愿进行协 调，或对 替代成本”型价值进行转化，使它们能在价值概念 上一致起来。

\section{6 复合型价值的可解析性}

前面提到, 为了计算某一物种的总价值, 需要对其中的 复合型价值分量进行分解，从而将这一物种的真正价值离析 出来。这就是复合型价值的可解析性。

在经济上,一个商品的价格,均可不失一般性地被视为 复合型价格, 因为它起码是由三大要素所决定, 它们是: 原材 料或中间物品、人力、资本。而且, 具有市场价格的商品, 其 价值是可解析的。可以不失一般性地将这一商品的价格函 数表示为:

$$
P=f\left(x_{m}, x_{e}, x_{c}\right)
$$

其中, $P$ 为价格 $f$ 为价格的函数关系, $x$ 为影响价格的要素; 下标 $m$ 为原料与中间物品 $e$ 为劳动力,$c$ 为资本投入。为确 定各要素对价格 $P$ 的影响, 可将式 (2) 改写成全微分形式 :

$$
\Delta P=\sum_{i=m e f} \frac{\partial f}{\partial X_{i}} \Delta X_{i}
$$

这样,要素 $x_{i}(i=m, e, c)$ 对价格 $P$ 的影响，或者说在 $P$ 中所 占的份额, 可以表示为:

$$
\lambda_{i}=\frac{\partial f}{\partial X_{i}} \frac{\Delta X_{i}}{\Delta P}
$$

其中 , $\lambda_{i}$ 为要素 $X_{i}$ 的价格影响度或影响份额。根据 $\lambda_{i}$ 可以 计算要素 $i$ 在商品中的体现的价值。

对于一个生物物种在群落层次与生态系统层次的作用 和影响理论上也可按照上述思路加以处理。只不过这时的 可解析函数, 大多不是描述群落层次与生态层次的价格函 数, 而是描述这些功能的函数。通过功能与价值之间的对应 关系, 可以将一个物种对群落层次与生态系统层次的功能影 响 转化为该物种对这些功能价值的影响。在生态学及其相 邻学科领域 (如地理学、气象学、土壤学等), 利用种群间的相 互作用 (捕食一猎食关系、共生关系、寄生关系、食物链网关 系等等) 来构成群落层次的功能函数 (主要是 Groot 所说的 生产功能), 以及利用群落与环境的相互作用 (能源流与物质 流) 来构成生态系统层次的功能函数 (主要是 Groot 所说的 “调节功能”与 承载功能”), 是较为可行的。由此, 将一个 生物物种在群落层次与生态系统层次功能中的影响离析出

\section{7 生物多样性价值评估的误差测算}

生物多样性价值评估, 与其他经济学量一样, 都存在误 差。误差大小影响着评估的可信性。对于生物多样性价值 评估,一如对于环境破坏的经济损失计算一样, 不同的研究 结果之间差异很大。如, 世界生态系统功能价值的低值和高 值分别为 $16 \times 10^{12} \sim 54 \times 10^{12}$ 美元, 中国大气污染损失的低 值和高值分别为 $15.1 \times 10^{9}$ 元 (美国东西方研究中心 1996 ) 与 $411 \times 10^{9}$ 元 (世界银行, 1997)。这说明, 影响包括生物多 样性在内的环境和生态价值评估的误差的原因, 远比一般经 济学理解复杂得多。

大致可以认为, 导致生物多样性价值计量差异的原因有 三个方面，即: 价值概念、计量方法、计量参数。

在价值概念方面，人们对生物多样性价值及其可计算性 的认识远不是一致的。根据 Pearce 等人的观点, 生物多样性 的总经济价值, 应由 4 部分构成; 而根据 Costanza 等人对世 界生态系统功能价值的处理, 仅涉及“直接使用价值”与间 接使用价值”两部分。很显然,Pearce 等人所定义的价值” 范畴远比 Costanza 等人宽泛得多。

在计量方法方面, 人们的认识差异更大。这不仅表现在 本文第 1 节所评论的 Pearce 与 Costanza 在世界生态系统功 能价值的计算方法之争, 同时也表现为本文在第 4 节所讨论 的计算方法的恰当性问题: 是基于市场价格,还是基于支付 意愿; 是坚持正面计算, 还是采用破坏损失或替代成本来评 估。上述处理方法对价值评估的结果是各不相同的。除此 而外, 还有其他一些方法学问题。如表 2 所列的旅游成本 法”与享用型价格法”, 它们试图从旅游支出的变化、工资 的变化、地价的变化来反映环境 (包括生物多样性) 变化的价 值影响。当在总价值计算中引入这两种方法时, 将会产生如 下问题: 第一, 它们既非完全独立, 又非完全等同, 因而如同 时采用, 会产生重复计算, 如择一而用, 又有漏算之虞; 第二, 这两种方法所产生的价值结果按说应是用于顶替一些 非经 济” 即与市场无关” 领域的价值分量的, 然而可以肯定, 它 们的结果远小于那些价值分量之和。

在计量参数方面, 同样差异很大。各国市场仍具有一定 的封闭性, 物品、人力、资本等生产要素并未真正自由地全球 流动的情况, 因此, 无论是市场价格还是 WTP , 都与当地或所 在国的经济社会发展水平密切相关。这意味着计量参数只 能做到国别统一或区域统一。而当将由此所得的结果置于 国际或全球尺度来考察时, 差异必然产生。

以上分析表明，在生物多样性价值评估方面的差异或误 差, 主要源自概念、方法、以及参数选择的不规范性。因此, 规范化是减少与消除生物多样性价值计算差异 (或误差) 的 首要条件。

为了强化对生物多样性价值计量的规范化, 这里提出如 下规则 : 
1) 凡是进入生物多样性价值计算的价值分量，必须是 可计算的。据此，一些不具备可计算性的价值分量，如涉及 “代际转移”与灭绝” 状态的 选择价值”与 存在价值”, 将 不在其列。也就是说，生物多样性价值计量仅计入生物多样 性在物种层次、种群层次、群落层次、生态系统层次的功能并 在人类 经济相关”领域和 非经济相关”领域中能以货币表 示的价值量。

2) 生物多样性在物种层次、种群层次、群落层次、生态 系统层次的功能的具体内容, 以及这些功能对人类 经济相 关”领域或 非经济相关”领域的影响（即价值分量类目）, 应 由国际生态学界与经济学界, 特别是由生态经济学与环境经 济学界达成共识。

3) 国际生态经济学与环境经济学界还应就各种价值分 量的计算方法，即价值参数的选型与计算途径的选择达成共 识; 特别是为了实现参数选型与计算途径选择的一致性，应 对不同参数类型之间和不同计算途径之间的转换建立某种 规则。

4) 国际生态经济学与环境经济学界还应就不同国家、 不同区域的生物多样性价值建立 汇率”标准，以实现这些价 值的国际换算与国际可比较性。

可以认为, 在上述四项规则得到真正、全面地落实之前， 生物多样性价值计算是很难取得充分的经济可信度的，也很 难在实际经济决策 (即政府决策、企业决策) 中真正发挥有效 影响。

\section{8 生物多样性破坏的经济损失计算 计算原则}

生物多样性破坏的经济损失计量, 无疑是生物多样性价 值理论应用的一项重要内容。然而，它的意义远不止于此。 生物多样性破坏的经济损失计算对于生物多样性价值, 有着 极为关键的认识论意义以及概念与方法学意义。在相当大 程度上可以说，人们是通过生物多样性破坏引起的经济损失 来认识生物多样性价值, 并创造对这一价值的计量方法的。 之所以这样说, 是出于以下两个原因。

原因一。由于生物多样性是先天存在的，它构成了人类 的生存背景，人们对它的功能和资产已熟视无睹。这与人类 对人造资产的功能和价值的认识迥然不同。在这种情况下， 人们大多不得不从生物多样性破坏一一或者更广义地说，从 人类生存背景的破坏一一对人类生存的威胁性影响，来认识 它们的功能和价值。

原因二。由于生物多样性如同人类生存的其他背景要 素一样 均为公共物品。它们与人造资产 (有着有限而明确 的权属关系, 并可经由市场交易进行权属转移) 不同, 其价值 难以直接正面地计量。对此, 人们大多是从计算其破坏引起 的形形色色的损失中发现，这些损失计算方法对于计算生物 多样性价值同样是有用的，或有启示性意义。

然而，如前所述，生物多样性破坏的经济损失与生物多
样性价值并不等同。而且, 生物多样性破坏的经济损失计 算, 至今仍不能说是规范的。这里着重讨论生物多样性破坏 损失计算中必然面临的两个关键问题, 同时也就解决这两个 问题, 提出应当遵循的原则。

问题 1 如何确认进入生物多样性破坏损失计算的生物 多样性破坏量量值

当人们处理 生物多样性破坏的经济损失”这一概念时， 总是不自觉地将它置于经济统计规则上来理解的, 即总是将 这一“损失”界定为年度损失。这是对的。但是如何计算这 “ 年度损失”, 则存在两种截然不同的认识。这里着重讨论 环境和生态破坏的损失计算截然不同的两种处理。

一种认识是, 年度损失应当基于当年造成的环境生态 破坏量” 来计算。可以将此称为年度破坏量”处理。它典 型地表达于下式中

$$
S S N N P=C+\Delta K_{m}-C_{D}-\Delta K_{n}
$$

其中 $S S N N P$ 为 可持续的社会净国民产值”, $C$ 为总消费， $\Delta K_{m}$ 为人造资产年度增量, $C_{D}$ 为抵御性消费, $\Delta K_{n}$ 为自然资 产年度增量 ${ }^{\mathbb{1}}$ 。这里值得注意的是 $\Delta K_{n}$, 它是反映着包括生 物多样性在内的自然资产破坏的损失, 而这种损失是以年度 破坏量 $\Delta K_{n}$ 来计算的。在国际和国内生态经济和环境经济 学界, 这一认识是较为流行的。

另一种认识是, 这一“年度损失”的计算, 不应当基于当 年造成的生物多样性破坏量 (即年度破坏量来计算) ,而应基 于一种 积累破坏量” 概念来计算。这 “ 积累量”可以表达 为下式

$$
S_{N}^{n}=\sum_{i=1}^{n} \Delta K_{N}^{i}=K_{N}^{o}-K_{N}^{n}
$$

式中 $S_{N}^{n}$ 为到第 $n$ 年为止自然资产 (同样适用于生物多样 性) 破坏的积累量 $\Delta K_{N}^{i}$ 为在第 1 年至第 $n$ 年时段中各年造 成的破坏量 (即年度破坏量) , $K_{N}^{o}$ 为基准年的自然资产存量， $K_{N}^{n}$ 为第 $n$ 年的自然资产存量。上式说明, 第 $n$ 年因自然资 产破坏造成的经济损失, 应取决于多年来的年度破坏量之和 (即积累破坏量)，而不是当年造成的破坏量。

本文坚持, 式 (6) 是恰当的, 而式 (5) 是不合理的。这是 因为自然资本与人造资本在经济学上的不同性质。人造资 产有着有限的价值, 这一价值或可由它的市场价格与其寿命 之积决定, 或可由其功能的市场价格与其寿命之积决定。由 于人造资产的寿命是有限的, 因而它的价值是有限的。对于 自然资产来说，形式上，它的价值仍可按上述方式决定，但由 于自然资产的寿命是无限的，因而它的价值亦是无限。很明 显, 人们不能以对具有有限价值的资产的处理方式来处理具 有无限价值的资产。如果坚持这样做，既会造成理论上的矛 盾, 又会造成实践中的谬误。这里试以一个假设性案例加以 说明。设有一块面积为 $100 \mathrm{hm}^{2}$ 的森林, 以每年 $10 \mathrm{hm}^{2}$ 速度

(1) 在严格的数学意义上, 在发生自然资产破坏的情况下, $\Delta K_{n}$ 应为 负值。因此在 $S S N N P$ 表达式中, 不应为 $-\Delta K_{n}$ ”, 而应为 $+\Delta K_{n}$ ”。 
递减，试分别计算其在第 1 年与第 10 年造成的水土流失损 失。如果依据式 (5) 则第 1 年与第 10 年的损失是一样的。 这显然是不合理的，因为第 10 年之前因森林破坏造成的水 土流失在第 10 年照样会发生，因而第 10 年的损失应为从第 1 年到第 10 年的破坏量之和。这正是式 (6) 表达的思想。

由此可以对进入破坏损失计算的生物多样性破坏量量 值, 提出一个确认原则, 它就是 累积破坏量”而不是 年度 破坏量”。

问题二: 如何确认物种破坏在群落层次和生态系统层 次造成的损失

这一问题主要与单物种破坏或少数物种破坏有关, 而且 主要与那些在群落和生态系统中具有基础性支持作用 (或处 于食物链网的关键性控制位置) 的物种的破坏有关。当这些 物种遭到破坏时, 其损失不仅在基因层次、种群层次, 而且在 群落层次与生态系统层次。由于这些物种在其中的基础性 支持作用, 因此, 它们的破坏对群落和生态系统的影响将是 关键性的, 甚至是颠覆性的。这样, 当计算这些物种破坏对 群落和生态系统功能造成的损失时, 不能以这些物种自身在 其中的价值损失来表示, 而应以整个群落和生态系统的功能 价值损失来表示。这就是本文在第 4 节所强调的,生物多样 性破坏的经济损失一定大于或等于它的自身价值。

由此可以对进入破坏损失计算的生物多样性功能量值 提出一个确认原则, 它应反映该物种对群落和生态系统功能 破坏的影响，而不是自身在其中的价值损失。

\section{9 对涉及生物多样性工程的投入一产出分析:CBA 与 CEA 应用框架}

生物多样性价值理论的另一个重要的应用领域, 是形形 色色与生物多样性有关的工程。这既包括生物多样性保护 工程 (如迁地保护、就地保护, 以及兼具一定经济目的的绿化 工程、荒漠化治理工程、湿地与海岸保护工程，等等），又包括 各种自然资源利用工程 (旅游区开发、大型水利工程建设、农 垦项目，等等)。长期以来，人们或是认为生物多样性保护工 程是公益性、非盈利事业, 无需进行经济学分析和处理, 或是 对涉及生物多样性的工程空泛笼统地提出经济效益、社会效 益、环境效益兼顾的口号, 而实际上不作具体分析地将它们 拢合在一起。这些认识与做法都是不对的。一项工程或一 项事业, 只要与钱打交道, 就是与经济学相关, 就必须对它的 投入和产出关系进行经济学分析。只有经过具体而严格的 经济分析，才能将经济效益、社会效益、环境效益既有区别又 有联系地统一起来。

对于涉及生物多样性工程的投入 - 产出关系分析, 主要 有两类方法。一类是成本 - 效率分析 (Cost-Benefit Analysis , 简称 $\mathrm{CBA}$ )，它主要用于对工程中具有经济、市场价值的事物 进行分析; 另一类是成本 - 效果分析 (Cost-Effectiveness Analysis，简称 CEA）,它主要用于对工程中具有非经济、非市场
意义的事物进行分析。一般来说, 涉及生物多样性的工程大 多都兼具经济的与非经济的目的和特征 (尽管各自份额随实 际情况而变化), 因此, 无论是 CBA 还是 CEA, 都是同时需要 的。其中, $\mathrm{CBA}$ 主要与工程的经济效益有关, $\mathrm{CEA}$ 主要与工 程的社会效益和环境效益有关。

为了将一个工程的经济效益、社会效益、环境效益三者 既有区别又有联系地统一起来, 对这一工程的投入 - 产出分 析, 可以不失一般性地分为三个层面进行, 即 : 经济层面、社 会环境层面、代际层面 (即可持续性发展层次)。

在经济层面, 分析应当是纯经济学的, 即 $\mathrm{CBA}$ 。它应当 紧紧围绕工程的经济目标和经济效果。这里 $\mathrm{CBA}$ 中的成 本, 是以货币表达的物品、人力和资本的总投入; $\mathrm{CBA}$ 中的效 益应是以货币表示的可以直接产生市场价值的总产出。从 理论上讲, 产出/投入之比越大, 其经济效果则越好。如果这 种 $\mathrm{CBA}$ 分析未能达到预期的经济目标或经济效果, 那么这 一工程在经济上是失败的。

在社会环境层面上, 分析应当是生态经济学或环境经济 学的。这时的分析空间已不只是工程本身, 而应包括工程可 能影响的地区 (即工程影响链的下游”地区), 这时涉及更 多的是一些体现经济外部性的事务。这时的分析方法应是 $\mathrm{CEA}$ 。这里 CEA 中的成本将不是工程成本, 而是工程所引起 的社会成本(如人口迁移, 因传统资源丧失而产生的职业变 化, 等等);CEA 中的效果 (effectiveness) 应是环境效益及其 对区内外 (包括下游地区) 的社会效果 (改善了生产和生活 条件)。对 CEA 的评价标准, 不能简单地沿用 CBA 的做法, 认为效果/成本之比愈大愈好, 而应关注成本与效果之间的 协调。这一分析的决策意义在于: 应使人们认识到,工程的 社会环境效果往往是以一些人的利益丧失或遭受损害为代 价的，因而，工程受益者应对受损者进行补偿。

在代际 (即可持续发展) 层面,一般无法在定量意义上进 行分析。但提出以下三项规则还是必要的和可行的, 即 : 1) 确保工程符合当代人的生态环境追求;2) 确保被改造生态 系统的可恢复性; 3) 确保所涉及的生物多样性永续生存而 不是濒于灭绝。

应当指出, 上述三个层次的分析, 各有侧重, 彼此相关, 但并不等同, 彼此不能替代。尤其对于经济层面与社会环境 层面, 人们往往喜欢以加总 (aggregative) 方式来表达总成本 与总效果, 这是不对的, 或者至少说是不恰当的。对于一个 具有明确的经济目标的生物多样性相关项目, 即使其加总形 式的产出/投入比很好, 但经济层面的 B/C 比不好, 那么这一 项目也是失败的。同样, 对于一项具有明确社会环境目标的 生物多样性相关工程, 即使其加总形式的产出/投入比很好, 但其社会环境层面的 $\mathrm{E}$ 与 $\mathrm{C}$ 并不协调, 那么这一项目也是不 可取的。可见, 没有分析的 加总” 只能混淆两个层次之间的 区别, 既不能准确地对每个层面的绩效做出判断, 又不能准 确地对每个层面绩效的改善做出决策。因此, 对于一个涉及 
生物多样性的工程，人们首先需要有针对性地具体分析，然 后再在不同层面之间进行综合协调。

\section{参考文献}

徐嵩龄, 1998. 生态资源破坏经济损失计量的概念与方法的 规范化. 见: 中国环境破坏的经济损失计量: 实例与理 论研究. 北京: 中国环境科学出版社, 121 154

Adger N, 1993. Sustainable national income and natural resource degradation in Zimbabwe. In: Turner R K( ed.), Sustainable Environmental Economics and Management: Principles and Practice, London: Belhaven

Costanza R, Ralph d'Arge, Rudolf de Groot, Stephen Farber, Monica Grasso, Bruce Hannon, Karin Limburg, Shahid Naeem, Robert V. O' neill, Jose Paruelo, Robert G. Raskin, Paul Sutton and Marjan van den Belt, 1997. The value of the world ecosystem services and natural capital, Nature, 389: 253

Costanza R, Serafy S E, Lutz E, 1998. A reply. Environment, $\mathbf{4 0}(2): 26 \sim 27$

de Groot R, 1992. Functions of Nature. Wolters-Noordhoff, Netherlands

Pearce D and Turner D, 1990. The Economics of Environment and Natural Resources. London: Earthscan

Daly H E, 1989. Toward a measure of sustainable social net national product. In: Ahmad Y J et al. (ed.), Environmental Accounting for Sustainable Development, World Bank, Washington D. C.

Pearce D, 1993. Economic Values and the Natural World, Appendix II , London: Earthscan

Pearce D, 1998. Auditing the Earth. Environment, 40(2): 23 28

Pearce D, 1998. Auditing the Earth. Environment, 40(2): 23 28

Pimm S, 1997. The value of everything. Nature, 387

Sagoff M, 1997. Can we put a price on nature's services? Philosophy and Public Policy, 17(3): 7

(责任编辑：时意专) 